\title{
Chromosome-scale assembly and evolution of the tetraploid Salvia splendens (Lamiaceae) genome
}

\author{
Kai-Hua Jia', Hui Liu', Ren-Gang Zhang², Jie Xu', Shan-Shan Zhou', Si-Qian Jiao', Xue-Mei Yan', Xue-Chan Tian', \\ Tian-Le Shi', Hang Luo', Zhi-Chao Li', Yu-Tao Bao', Shuai Nie', Jing-Fang Guo', Ilga Porth ${ }^{3}$, Yousry A. El-Kassaby ${ }^{4}$, \\ Xiao-Ru Wang ${ }^{1,5}$, Charles Chen ${ }^{6}$, Yves Van de Peer ${ }^{7,8,9,10}$, Wei Zhao ${ }^{1,5 凶}$ and Jian-Feng Mao $\mathbb{1}^{1 凶}$
}

\begin{abstract}
Polyploidization plays a key role in plant evolution, but the forces driving the fate of homoeologs in polyploid genomes, i.e., paralogs resulting from a whole-genome duplication (WGD) event, remain to be elucidated. Here, we present a chromosome-scale genome assembly of tetraploid scarlet sage (Salvia splendens), one of the most diverse ornamental plants. We found evidence for three WGD events following an older WGD event shared by most eudicots (the $\gamma$ event). A comprehensive, spatiotemporal, genome-wide analysis of homoeologs from the most recent WGD unveiled expression asymmetries, which could be associated with genomic rearrangements, transposable element proximity discrepancies, coding sequence variation, selection pressure, and transcription factor binding site differences. The observed differences between homoeologs may reflect the first step toward sub- and/or neofunctionalization. This assembly provides a powerful tool for understanding WGD and gene and genome evolution and is useful in developing functional genomics and genetic engineering strategies for scarlet sage and other Lamiaceae species.
\end{abstract}

\section{Introduction}

Lamiaceae or Labiatae, or the mint family, is one of the largest families within the flowering plants, with 236 genera and more than 7000 species $^{1}$. Plants in the mint family are chemically diverse, of great ecological, economic, and cultural importance, and extensively cultivated because of their ornamental value, flavor, fragrance, and medicinal properties. Composed of approximately 1000 species $^{1}$, the genus Salvia is the largest genus of the mint family that seems to be polybasic, with different species having polyploid origins ${ }^{2}$. Tetraploid

\footnotetext{
Correspondence: Wei Zhao (zhaowei.nice@gmail.com) or Jian-

Feng Mao (jianfeng.mao@bju.edu.cn)

${ }^{1}$ Beijing Advanced Innovation Center for Tree Breeding by Molecular Design, National Engineering Laboratory for Tree Breeding, Key Laboratory of Genetics and Breeding in Forest Trees and Ornamental Plants, Ministry of Education, College of Biological Sciences and Technology, Beijing Forestry University, Beijing 100083, China

${ }^{2}$ Ori (Shandong) Gene Science and Technology Co., Ltd, Weifang 261000 Shandong, China

Full list of author information is available at the end of the article

These authors contributed equally: Kai-Hua Jia, Hui Liu, Ren-Gang Zhang, Jie Xu
}

$(2 n=4 x=44)$ scarlet sage (Salvia splendens or tropical sage $)^{3}$ is among the most commonly cultivated ornamental plants and is characterized by dense flowers, wide color variation, long-lasting flowering (longer than 2 months), and resistance to pests and diseases ${ }^{4}$. S. splendens is a worldwide popular bedding plant with significant social and economic value ${ }^{5}$. Notwithstanding these virtues, genomic resources are only available for very few mint species, which severely limits further evolutionary and functional studies.

Polyploidization, also known as whole-genome duplication (WGD), is widespread across land plants and particularly frequent in ferns and angiosperms ${ }^{6,7}$, generating novel and varied phenotypes ${ }^{8-10}$. WGD events can result in instant reproductive isolation, as the difference in chromosome number impedes reproduction, promoting speciation, evolution, and biodiversity ${ }^{11,12}$. Polyploidization can enhance adaption, as it has been associated with survival in stressful environments ${ }^{13}$ (e.g., within aspen's southwestern distribution in North America)

\section{(c) The Author(s) 2021}

(c) (i) Open Access This article is licensed under a Creative Commons Attribution 4.0 International License, which permits use, sharing, adaptation, distribution and reproduction cc) in any medium or format, as long as you give appropriate credit to the original author(s) and the source, provide a link to the Creative Commons license, and indicate if changes were made. The images or other third party material in this article are included in the article's Creative Commons license, unless indicated otherwise in a credit line to the material. If material is not included in the article's Creative Commons license and your intended use is not permitted by statutory regulation or exceeds the permitted use, you will need to obtain permission directly from the copyright holder. To view a copy of this license, visit http://creativecommons.org/licenses/by/4.0/. 
Table 1 Statistics of the S. splendens genome assembly.

\begin{tabular}{lll}
\hline Assembly feature & V1.0 & V2.0 \\
\hline Assembly size (Mb) & 809.2 & 807.7 \\
Anchored size (Mb) & - & 767.09 \\
Number of contigs & 2204 & 1655 \\
Max. contig length (Mb) & 10.81 & 12.92 \\
Contig N50 length (Mb) & 2.27 & 3.77 \\
Contig N90 length (Mb) & 0.27 & 0.59 \\
Number of scaffolds & 1525 & 1184 \\
Max. scaffold length (Mb) & 12.94 & 47.13 \\
Scaffolds N50 length (Mb) & 3.12 & 35.13 \\
Scaffolds N90 length (Mb) & 0.43 & 25.62 \\
GC content (\%) & 38.84 & 38.84 \\
Repeat region \% of assembly & 57.52 & 56.94 \\
Predicted gene models & 54,008 & 88,489 \\
\hline
\end{tabular}

(Goessen, Isabel, Giguère, Gros-Louis, Touchette, Laroche, Boyle, Lamothe, Tischenko, Soolanayakanahally, Mock, Bousquet, Hernández Velasco, Simental Rodriguez, Wehenkel, Porth (2021) in prep. How perennial angiosperms cope with environmental constraints: adaptive genetic variation and plasticity of life-history traits in Populus tremuloides). Gene duplications result in genetic redundancy, thereby increasing genetic resources and masking deleterious mutations through compensation. Polyploidization and initial redundancy also offer new possibilities for gene evolution: one copy can be degraded, both copies can be conserved by dosage balance, or their expression patterns may diverge (tissue-specific expression through subfunctionalization ${ }^{14}$ or even the evolution of new functions through neofunctionalization ${ }^{15,16}$ ). In such polyploids, the simultaneous duplication of many genes provides extra genetic material on which evolution can work, increasing genetic variation, which is considered an important mechanism for evolving adaptive traits $^{10,16}$. However, although polyploidization may be significant in increasing genetic variation and affecting gene expression, we still have a limited understanding of the full extent of whether homoeologs resemble or differ from each other in their expression patterns, the spatiotemporal dynamics of these relationships, and how genomic rearrangement, transposable element (TE) proliferation, and sequence diversification impact these differences.

A draft genome sequence for $S$. splendens has been published previously ${ }^{17}$, but scaffold-level assembly complicates deeper investigation of polyploidy and homoeolog divergence and evolution. Here, based on long-read sequencing and the $\mathrm{Hi}-\mathrm{C}$ scaffolding strategy, an
$807 \mathrm{Mb}$ chromosome-scale assembly was constructed for the tetraploid S. splendens, with $95 \%$ of the assembly anchored to 22 pseudochromosomes. We detected remnants of older WGD events and examined gene expression differences between homoeologs and potential associations with sequence diversification, genomic rearrangement, and TE proliferation. This genome assembly is essential to better understand the dynamic evolution following WGD in Lamiales; in addition, our findings will further benefit the functional genomics and molecular breeding of S. splendens.

\section{Results \\ "Gold standard" tetraploid genome assembly and annotation}

An elite tetraploid S. splendens cultivar, "aoyunshenghuo (Olympic flame)," developed through multiple rounds of selfing, was selected for sequencing. A total of 66 Gigabases (Gb) (82 X) of PacBio single-molecule long reads (average read length of $7.4 \mathrm{~kb})$ and $37 \mathrm{~Gb}(40 \mathrm{X})$ Illumina paired-end reads were generated for initial assembly ${ }^{17}$ (Supplementary Table 1). A primary assembly with contig and scaffold N50 values of 2.27 and $3.12 \mathrm{Mb}$ (Table 1, V1.0) was gained for further scaffolding after rounds of assembly, polishing, and comparison $^{17}$. To anchor the scaffolds to chromosomes, we constructed high-throughput chromosome conformation capture (Hi-C) libraries of $S$. splendens, generating $85 \mathrm{~Gb}$ (105 X) Hi-C paired-end reads (Supplementary Table 1). As a result, the contig and scaffold N50 of the final assembly were upgraded to 3.77 and $35.13 \mathrm{Mb}$, with $47.13 \mathrm{Mb}$ as the longest scaffold (Table 1, V2.0 and Supplementary Table 2). The final reference assembly comprised 22 chromosomescale pseudomolecules representing $95 \%$ of the $807 \mathrm{Mb}$ assembly (the pseudomolecules are hereafter referred to as chromosomes, Fig. 1 and Table 1). By filtering reads that showed sequence similarity to known chloroplast and mitochondrial genomes, we were able to assemble the chloroplast and mitochondrial genomes into single contigs with lengths of 150,607 and 347,308 bp, respectively (Supplementary Figs 1 and 2). The high-fidelity assembly was supported by a high ten-fold minimum genome coverage of 98.58\% (PacBio) and high mapping rates of 99.06\% (Illumina). Protein-coding regions were assembled to near completeness judged by the high Benchmarking Universal Single Copy Orthologs recovery of $92 \%$ in querying the embryophyte datase ${ }^{18}$ (Supplementary Table 3). Repetitive sequence regions were assembled to high continuity by a high long terminal repeat (LTR) assembly index score of 27.49 , reaching the "gold standard" 19 . These results suggest a highly accurate, very contiguous, and near-complete assembly.

We identified a total of 88,489 gene models, including 56,267 protein-coding genes, 28,993 long noncoding RNAs (lncRNAs), 1541 transfer RNAs (tRNAs), 


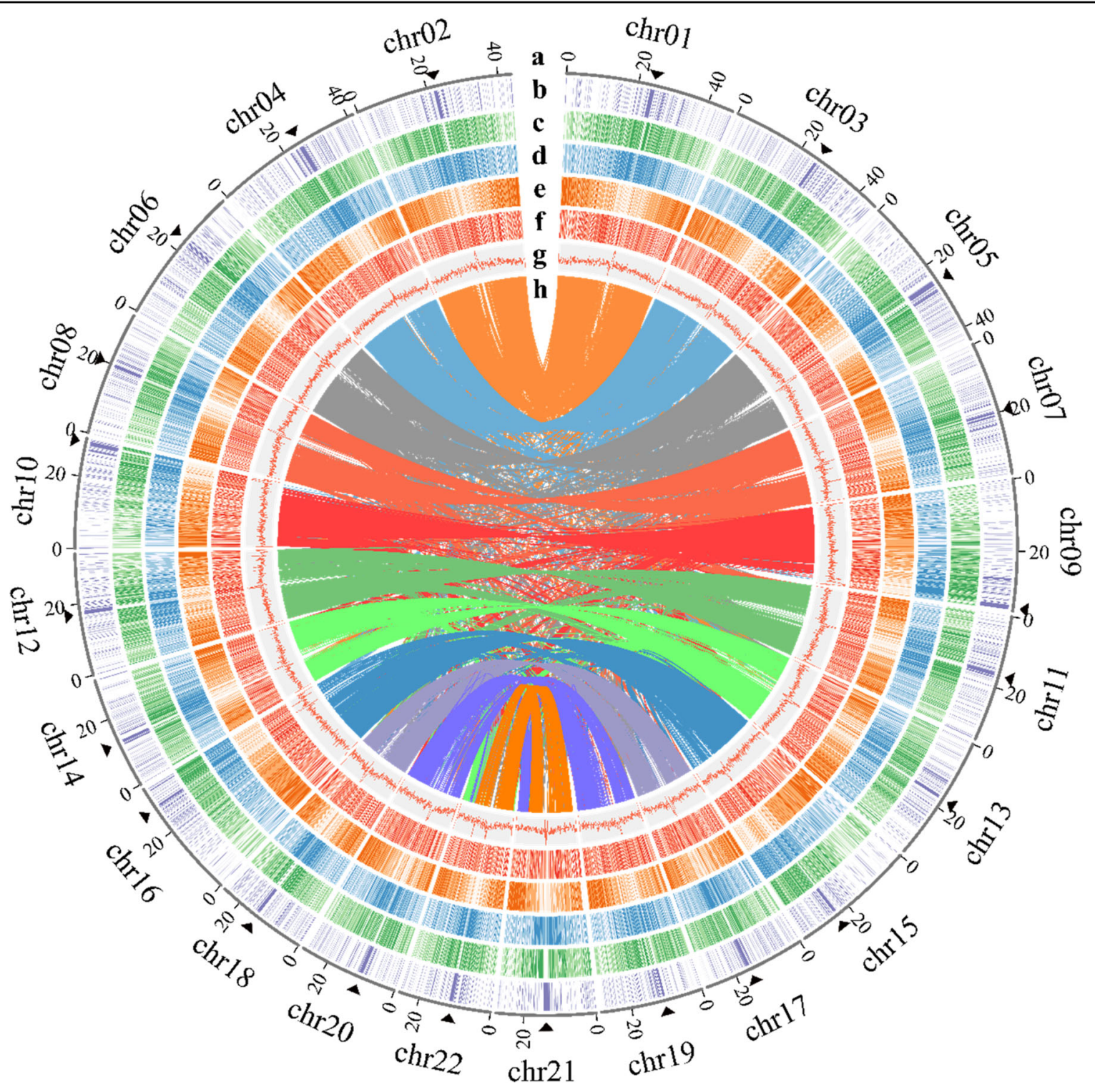

Fig. 1 Synteny and distribution of features in the S. splendens genome. Chromosome numbers are indicated on the outside. Within the circle plot, the darker the color is, the higher the density. a Length (Mb) of chromosomes and position of the pericentromeric regions (black triangles), b LINE1 (long interspersed nuclear elements 1) density, c Copia density, d Gypsy density, e Gene density, f Pseudogene density, g GC (guaninecytosine) content, and $\mathbf{h}$ Syntenic blocks

518 ribosomal RNAs (rRNAs), and 1170 extra unclassifiable noncoding RNAs (ncRNAs) (Table 1 and Supplementary Table 4). There were 53,771 protein-coding genes $(95.6 \%)$ that could be assigned to 22 chromosomes, and the gene density was highly skewed toward the distal region of chromosome arms (Fig. 1 and Supplementary Figs 3-24). Numerous transcription factor (TF; 3717) genes were predicted and classified into 56 gene families, including the major families bHLH, ERF, MYB, C2H2, NAC, and MYB_related, which contained 325, 321, 281, 221, 192, and 163 genes, respectively (Supplementary Table 5).

\section{Comparative phylogenomics and palaeopolyploidization}

To assess the palaeohistory of the Lamiales, we performed comparative genomic analyses incorporating S. splendens along with 12 Lamiales genomes and one outgroup (Vitis vinifera) (Fig. 2b, see Methods). Out of the 18,497 gene families (consisting of 364,467 genes), 557 genes from 88 gene families were found to be unique in the $S$. splendens genome (Supplementary Fig. 25). These unique genes are mainly related to plant resistance, such as the detection of biotic stimuli, hydrogen peroxide metabolism regulation, and salicylic acid biosynthesis (Supplementary Table 6). 


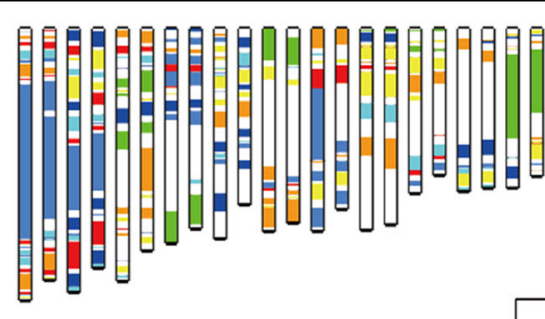

a

AEK chromosome: 1-7

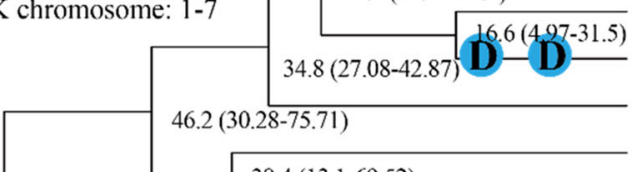

D

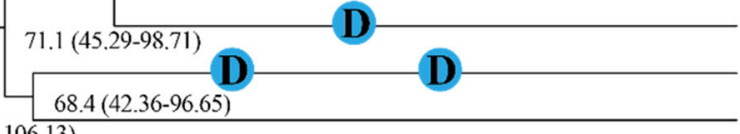

$83.4(54.82-106.13)$

$92.8(62.84-113.09)-\mathrm{T}$

$\mathbf{T}$

116.3

$103.7(70.79-120.21)$

D

D

25

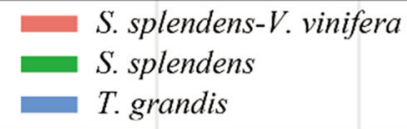

c

100

75

50

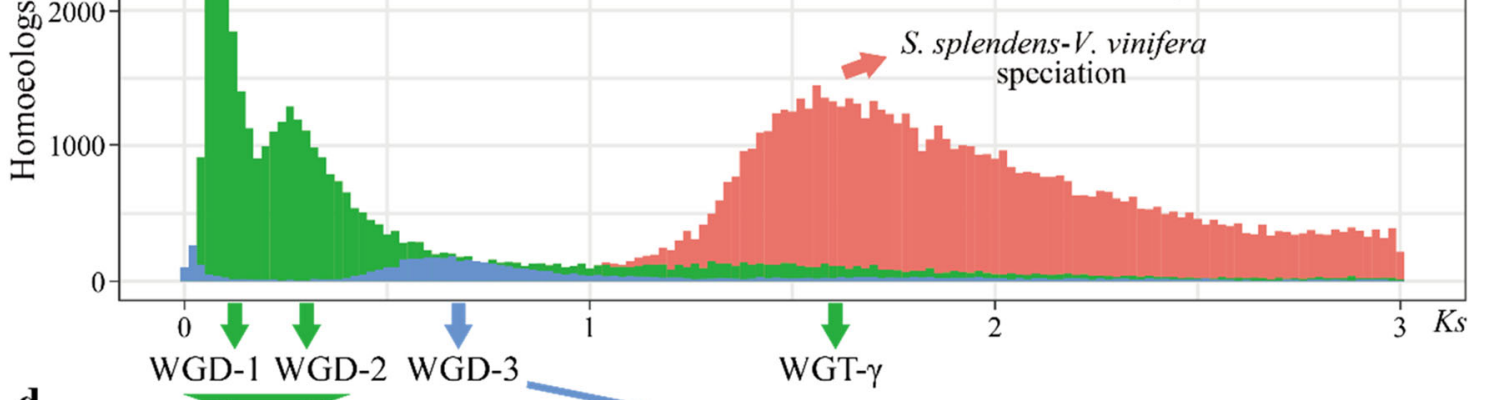

d

WGD-1 WGD-2 WGD-3

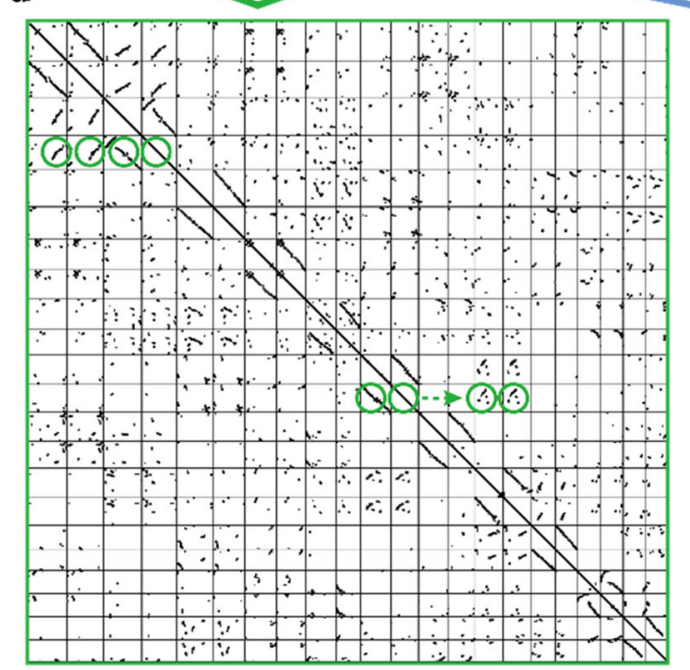

S. splendens genome

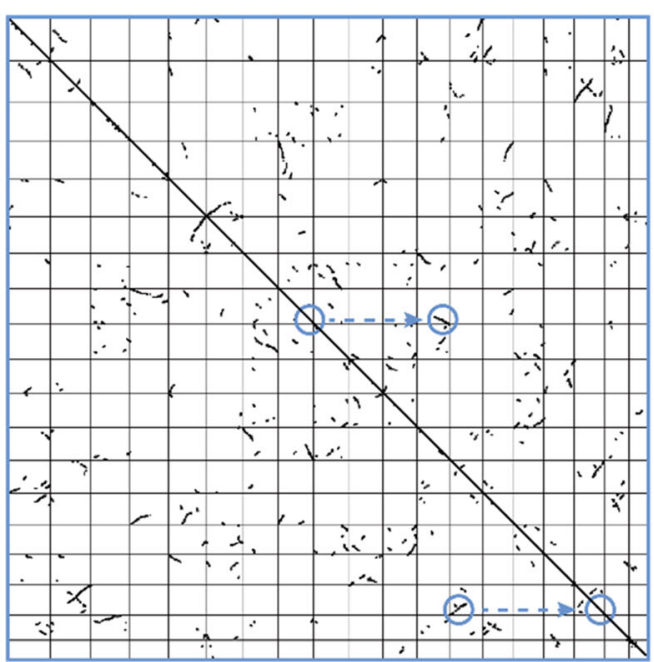

T. grandis genome

Fig. 2 S. splendens evolutionary history. a Comparison with ancestral eudicot karyotype (AEK) chromosomes reveals synteny. The syntenic AEK blocks are painted onto S. splendens chromosomes. b Inferred phylogenetic tree, divergence time, whole-genome duplication (WGD), and wholegenome triplication (WGT). c Ks distribution. y axis, S. splendens paralogs (green), T. grandis paralogs (blue), and S. splendens - V. vinifera orthologs (red). Polyploidization (WGD-1, WGD-2, WGD-3 and WGT-y) events are referenced on the $x$ axis. $\mathbf{d}$ Dot plots of paralogs in S. splendens and T. grandis that illustrate WGD-1, WGD-2 (1:4 chromosomal relationships in green circles), and WGD-3 (1:2 chromosomal relationships in blue circles) events 
Construction of a phylogenetic tree from 373 orthogroups confirmed the evolutionary relationship within Lamiales, and the divergence between S. splendens and Salvia miltiorrhiza was estimated at 16.6 MYA (million years ago) (Fig. 2b).

Ancient polyploidization is widespread in most plant lineages, providing a powerful resource for novel genes and the evolution of new gene functions, as well as the emergence of new species ${ }^{20}$. Comparison of S. splendens with an ancestral eudicot karyotype genome ${ }^{21}$ and intragenomic homology supported WGD-1 (which led to sage's tetraploid state) (Figs 1 and 2a). The $K s$ (the number of substitutions per synonymous site) distribution of intragenomic paralogs further showed two clear peaks of duplicate genes at $K s$ values of approximately 0.08 (WGD-1) and 0.26 (WGD-2) (Fig. 2c). The synteny analysis consistently revealed that these inferred WGD events (WDG-1 and WGD-2) in the $S$. splendens genome (Fig. 2d). Homoeology analysis of 11 pairs of homoeologous chromosomes indicated that they were highly conserved, despite few major rearrangements (Fig. 2d). Of the 53,771 protein-coding genes, $72 \%(40,246)$ were organized in macrosynteny, that is, still present at their ancestral genomic position and in intervals with a highly conserved gene order (Fig. 2d). The 1:4 syntenic relationship indicates that S. miltiorrhiza and Tectona grandis did not share these two WGD events (WGD-1 and WGD-2) with S. splendens (Supplementary Figs 26 and 27).

As shown in Fig. 2c, a peak near $K s=0.6-0.8$ (WGD-3) was identified by examining the intragenomic homology with Mimulus guttatus ${ }^{22}$, Sesamum indicum ${ }^{23}$, Antirrhinum majus ${ }^{24}$, Tectona grandis ${ }^{25}$, Boea hygrometrica ${ }^{26}$, and Utricularia gibba ${ }^{27}$. However, the Ks peak was not evident in S. splendens (Fig. 2c), which could be indicative of an accelerated substitution rate in S. splendens.

When analyzing the 1:1 syntenic relationship between $T$. grandis and Scutellaria baicalensis, M. guttatus, and $S$. indicum, the presence of the shared WGD-3 event emerged (Supplementary Figs 28-30). By combining phylogenetic analyses, we inferred that Andrographis paniculate, Handroanthus impetiginosus, S. indicum, M. guttatus, S. miltiorrhiza, S. baicalensis, T. grandis, and S. splendens all collectively shared WGD-3 (Fig. 2b). The 2:1 syntenic relationship between Andrographis paniculata and S. indicum revealed that, in addition to the WGD-3 event, $A$. paniculata might have experienced another independent WGD event (Supplementary Fig. 31). Together with the shared WGD found previously for $U$. gibba and $M$. guttatus ${ }^{27}$, these results collectively implicated that WGD-3 is a shared event among species of Lamiaceae (T. grandis, S. baicalensis, $S$. miltiorrhiza, S. splendens), Phrymaceae (M. guttatus), Pedaliaceae ( $S$. indicum), Bignoniaceae ( $H$. impetiginosus), Acanthaceae (A. paniculate), Lentibulariaceae ( $U$. gibba), and Linderniaceae (Lindernia brevidens) (Fig. 2b).
Subsequently, we examined whether $A$. majus shared this WGD-3 with these species. Syntenic analysis showed that there was no 1:1 syntenic relationship supporting the same WGD-3 event shared between A. majus, S. indicum, and T. grandis (Supplementary Figs 32 and 33). In contrast, 3:2 syntenic blocks were identified between A. majus, S. indicum, and T. grandis (Supplementary Figs 32 and 33), suggesting that $A$. majus did not share the WGD-3 event with these species. Unexpectedly, 3:2 syntenic block support suggests that $A$. majus may have undergone a wholegenome triplication event rather than a WGD ${ }^{24}$.

\section{Recent accumulation of LTR-RTs and LINE1-dominated pericentromeres}

Through an integrative approach, we identified 56.94\% of the assembly as repeat elements (Supplementary Table 7). The long terminal repeat retrotransposons (LTR-RTs) form the largest proportion (26.66\%) of repeats, while the most abundant LTRs were Gypsy elements, making up $17.59 \%$ of the genome, followed by Copia elements (8.70\%; Fig. 1 and Supplementary Table 7). Gypsy and Copia families of repeats were found to have significantly contracted in S. splendens compared to S. miltiorrhiza (29.83\%/Gypsy and $14.77 \% /$ Copia of the genome $)^{28}$.

For full-length LTR-RTs, significant differences in individual counts, average length, and genomic coverage were present in the Gypsy and Copia superfamilies. Most LTRRTs gradually accumulated during the last 5 million years, rather than showing accumulation by sudden bursts (Supplementary Fig. 34), possibly due to the relaxed purifying selection on TE overaccumulation after the last WGD (WGD-1 ${ }^{29}$. Abundant LTR-RTs overlapped with genes (Supplementary Figs 35 and 36), signifying the potential impacts of LTR-RTs on protein-coding genes. When comparing $S$. splendens to other Lamiales species with respect to LTR-RT accumulation and removal rates, we found that the S. splendens was characterized by large numbers of intact, solo, and truncated LTR-RTs and relatively low removal rates (Supplementary Fig. 37). These results implied that the recent expansion of LTR-RTs played a significant role in the genome size evolution of $S$. splendens.

Based on the $\mathrm{Hi}-\mathrm{C}$ interaction, the pericentromeric position was identified for each chromosome using an established pipeline $^{30}$ (Fig. 1 and Supplementary Figs 324 and 38). Interestingly, pericentromeric regions of $S$. splendens were enriched with long interspersed nuclear elements 1 (LINE1), along with an elevated guaninecytosine (GC) content (Fig. 1 and Supplementary Figs 324). Therefore, we further defined the pericentromeric regions by examining LINE1 density and found that the size of centromeric regions ranged from 0.3 to $1.2 \mathrm{Mb}$ and was delimited by 93-573 LINE1 elements (Supplementary Figs 3-24 and Supplementary Table 8). 
Structural variation between homoeologous chromosomes

Genomic structural variations (SVs) and/or rearrangements may occur through nonhomoeologous chromosome recombination between repetitive elements ${ }^{31}$. To test whether TEs are related to an increased rate of chromosome SVs, we first used the longest homoeologous chromosome as the reference and the shorter chromosome as a query to detect SVs between homologous chromosomes. In total, we identified 8036 large SVs, including 3053 duplications, 167 inversions, 865 translocations, 3160 inverted duplications, and 791 inverted translocations (Supplementary Figs 39 and 40 and Supplementary Tables 9 and 10). Furthermore, we randomly selected $10004 \mathrm{~kb}$ regions from each chromosome to compare the $2 \mathrm{~kb}$ regions upstream and downstream of SV breakpoints, and the results showed that TEs were significantly enriched within breakpoint regions (Supplementary Fig. 41). The TE content within breakpoint regions is nonrandom, with LTR being the most strongly enriched relative to other types of repeated elements (Supplementary Table 11). Our results suggest that TE insertions may provide substrates for $S$. splendens genome SV events.

In addition, we examined whether these different types of SVs have the same effects on the gene expression of homoeologs. Therefore, we identified genes related to SVs. A total of 18,031 genes were within the SV regions, of which $15,878(88.06 \%)$ were in syntenic blocks shared between homoeologous chromosomes (Supplementary Table 12). Furthermore, we investigated genes that showed $\geq 2$-fold significantly differential expression $(p<$ 0.05 ) on homoeologous chromosomes in a pooled analysis by combining data across all RNA-seq samples (root, stem, leaf, calyx, and corolla for red/purple varieties; Supplementary Table 1). Inverted duplications (62.58\%) and duplications $(56.25 \%)$ had a greater impact on differentially expressed homoeologs than translocations (48.54\%), inverted translocations (45.57\%), and inversions (44.84\%) (Supplementary Table 12). These results indicate that duplications had a significant effect on differential gene expression compared with other SVs, which may be because duplications can increase gene redundancy and render homoeologs more susceptible to mutation accumulation due to relaxed functional constraints on additional gene copies $^{32,33}$.

\section{Spatiotemporal expression patterns of homoeologs}

In polyploids, the quantitative variation of many traits is regulated by genetic interactions. Among the 16,495 highconfidence homoeologs between homoeologous chromosome pairs (see Methods), 7481 (45.35\%) exhibited significant $\geq 2$-fold differential expression in the pooled tissue. Interestingly, we found that various homoeologous chromosome gene pairs showed different expression differences (Fig. 3a). Among the 11 homoeologous chromosome pairs, the number of two-fold expressed genes did not show significant differences in five pairs (chr01 vs. chr02; chr03 vs. chr04; chr09 vs. chr10; chr13 vs. chr14; and chr15 vs. chr16) of homoeologous chromosomes, while the remaining pairs of homoeologous chromosomes (chr05 vs. chr06; chr07 vs. chr08; chr11 vs. chr12; chr17 vs. chr18; chr19 vs. chr20; and chr21 vs. chr22) did show significant two-fold expression differences (Fig. 3a, $\chi^{2}$ test, $p<0.05)$. Unbalanced homoeologous expression bias was also seen for different varieties and tissues (Supplementary Fig. 42).

Based on whether or not homoeolog expression bias was detected and dependent on the number of highly expressed genes between homoeologous chromosomes (Fig. 3a), we separated all 22 S. splendens chromosomes into the following four groups: (1) SA (significant bias and more highly expressed genes, chr05; chr08; chr12; chr18; chr19; and chr22); (2) SB (significant bias and fewer highly expressed genes, chr06; chr07; chr11; chr17; chr20; and chr21); (3) SC (no significant bias and more highly expressed genes, chr02; chr03; chr09; chr13; and chr15); and (4) SD (no significant bias and fewer highly expressed genes, chr01; chr04; chr10; chr14; and chr16). We then investigated the impact of the flanking $\mathrm{TE}$ on the gene expression of the homoeologs. To do so, four categories were created: (1) SAyes_SByes, with both homoeologs having TEs within $2 \mathrm{~kb}$; (2) SAyes_SBno, with the group SA genes having TEs within $2 \mathrm{~kb}$ and SB genes not; (3) SAno_SByes, with the group SB genes having TEs within $2 \mathrm{~kb}$ and SA genes not; and (4) SAno_SBno, where neither SA nor SB have TEs within $2 \mathrm{~kb}$. The numbers of genes that exhibited significant two-fold expression changes in these four categories are shown in Fig. 3b. Group SA chromosomes exhibited significant expression dominance over group SB chromosomes when both SA- and SB-type genes had TEs within $2 \mathrm{~kb}$ (SAyes_SByes category). When comparing both chromosome groups, and when TEs were not in proximity to a gene (SAyes_SBno category), the number of dominantly expressed genes was slightly higher than when TEs were near genes (Fig. 3b). Furthermore, we performed a similar analysis on chromosome groups SC and SD that lack homoeolog expression bias. For gene categories such as those detailed above (SCyes_SDyes; SCyes_SDno; SCno_SDyes; and SCno_SDno), no significant differences were found (Fig. $3 \mathrm{~b}$ ). These results indicate that TEs affect the homoeologous expression bias pattern between homoeologous chromosomes.

Based on the level of homoeologous gene differential expression, we defined the following four homoeologous expression bias categories: (1) a lower homoeolog expression-difference with FC (fold-change) $\leq|2|$ ( $p \leq$ 0.05), (2) a medium homoeolog expression-difference with $|2|<\mathrm{FC}<|8| \quad(p \leq 0.05)$, (3) a higher homoeolog 


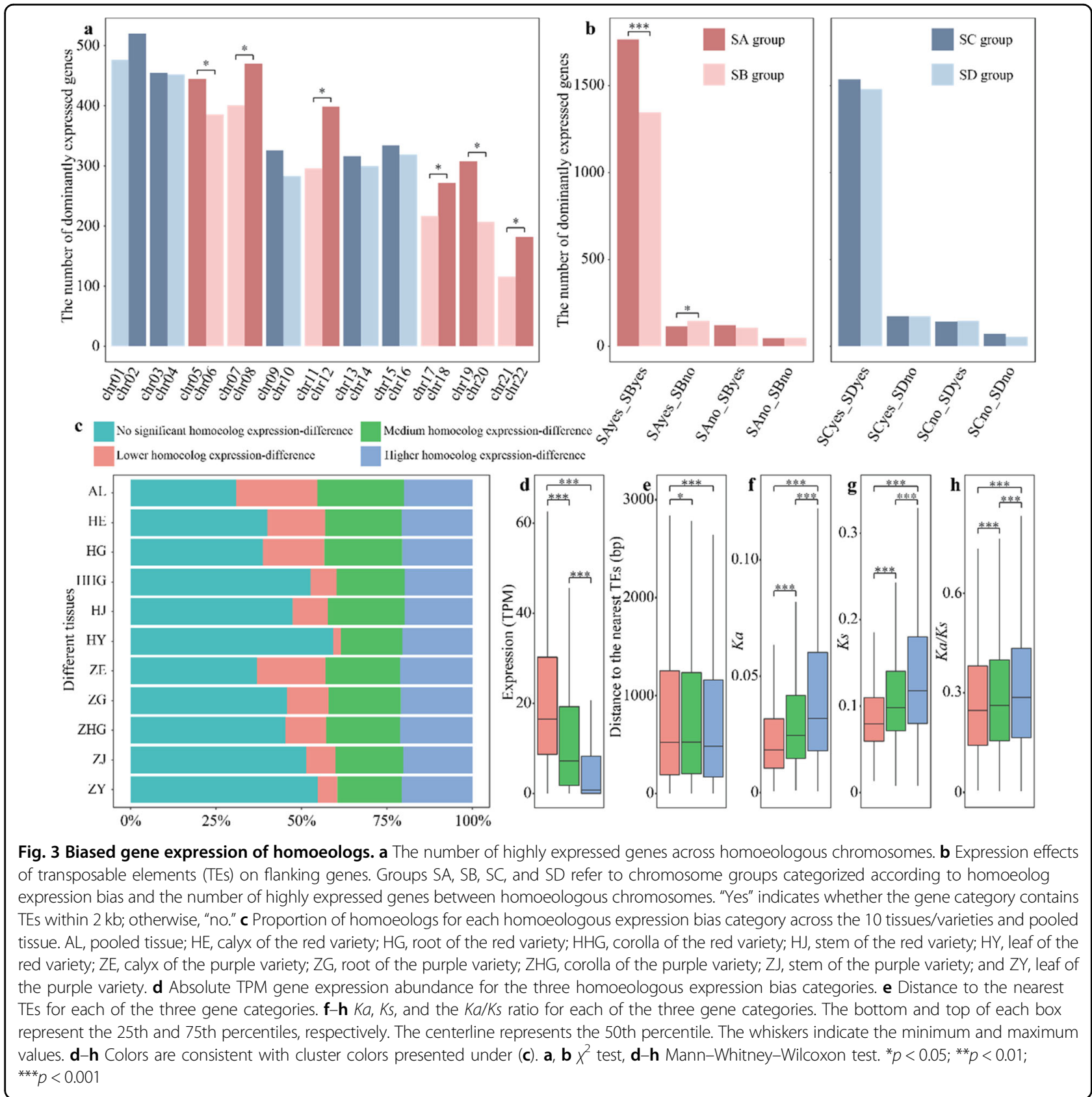

expression-difference with $\mathrm{FC} \geq|8|(p \leq 0.05)$, and (4) no significant homoeolog expression-difference $(p>0.05)$ categories. The differences in expression between most homoeologs were not significant, and the lower homoeolog expression-difference category showed a more dynamic change between different tissues, ranging from $2.3 \%$ in the leaf of the red cultivar (HY) to $23.8 \%$ in calyx of the purple cultivar (ZE) (Fig. 3c). We found that genes in the lower homoeolog expression-difference category were expressed across a wider range of tissues and had higher absolute transcript abundance (mean, 27.17 TPM) than genes in the medium expression-difference (mean,
21.68 TPM) and higher homoeolog expression-difference categories (14.56 TPM) (Fig. 3d). Absolute transcript abundance datasets indicated that the gene expressiondifference between homoeologs could not be attributed to the increased expression of homoeologs but to the relatively low expression of a specific homoeolog. Next, we examined the associations of TEs with the relative expression of homoeologs and found that genes in the higher homoeolog expression-difference category were closer to the flanking TE than those in the medium homoeolog expression-difference and lower homoeolog expression-difference categories (Fig. 3e). We also 
compared the nonsynonymous $(K a)$ and synonymous $(K s)$ substitution rates between homoeologs and observed that genes in the higher homoeolog expression-difference category had higher $\mathrm{Ka}, \mathrm{Ks}$, and $\mathrm{Ka} / \mathrm{Ks}$ than those in the medium homoeolog expression-difference and lower homoeolog expression-difference categories (Fig. 3f-h).

Polyploidy may confer phenotypic plasticity through homoeologs being differentially expressed among tissues and/or environmental conditions ${ }^{16}$. Therefore, we examined whether homoeologs retain their biased expression in different tissues (root, stem, leaf, calyx, and corolla for red/purple flower varieties; Supplementary Table 1). We found that most of the homoeologs remained in the original grouping, with only $0-19.81 \%$ (0-777 homoeologs) changing in at least one tissue (Fig. 4a). Adjacent categories (i.e., lower homoeolog expression-difference to medium homoeolog expression-difference or medium homoeolog expression-difference to higher homoeolog expression-difference) most often shifted to each other (1.91-19.81\%, 80-777 homoeologs), with just $0-0.84 \%$ (0-33 homoeologs) of categories moving across categories (i.e., lower homoeolog expression-difference to higher homoeolog expression-difference) (Fig. 4a). These data indicate that homoeologs are usually expressed stably in different tissues.

Based on the dynamic change of differential expression between the single tissue and pooled tissue samples, we focused on the $10 \%$ most stable homoeologs with consistently stable expression and the $10 \%$ most dynamic homoeologs that show the highest variable expression in different tissues/varieties. Stable homoeologs had higher expression and higher expression widths and were expressed in almost all samples, while dynamic homoeologs were more tissue-specific (Fig. 4b). "Stable" homoeologs were enriched for gene ontology (GO) terms associated with RNA splicing, mRNA processing, and

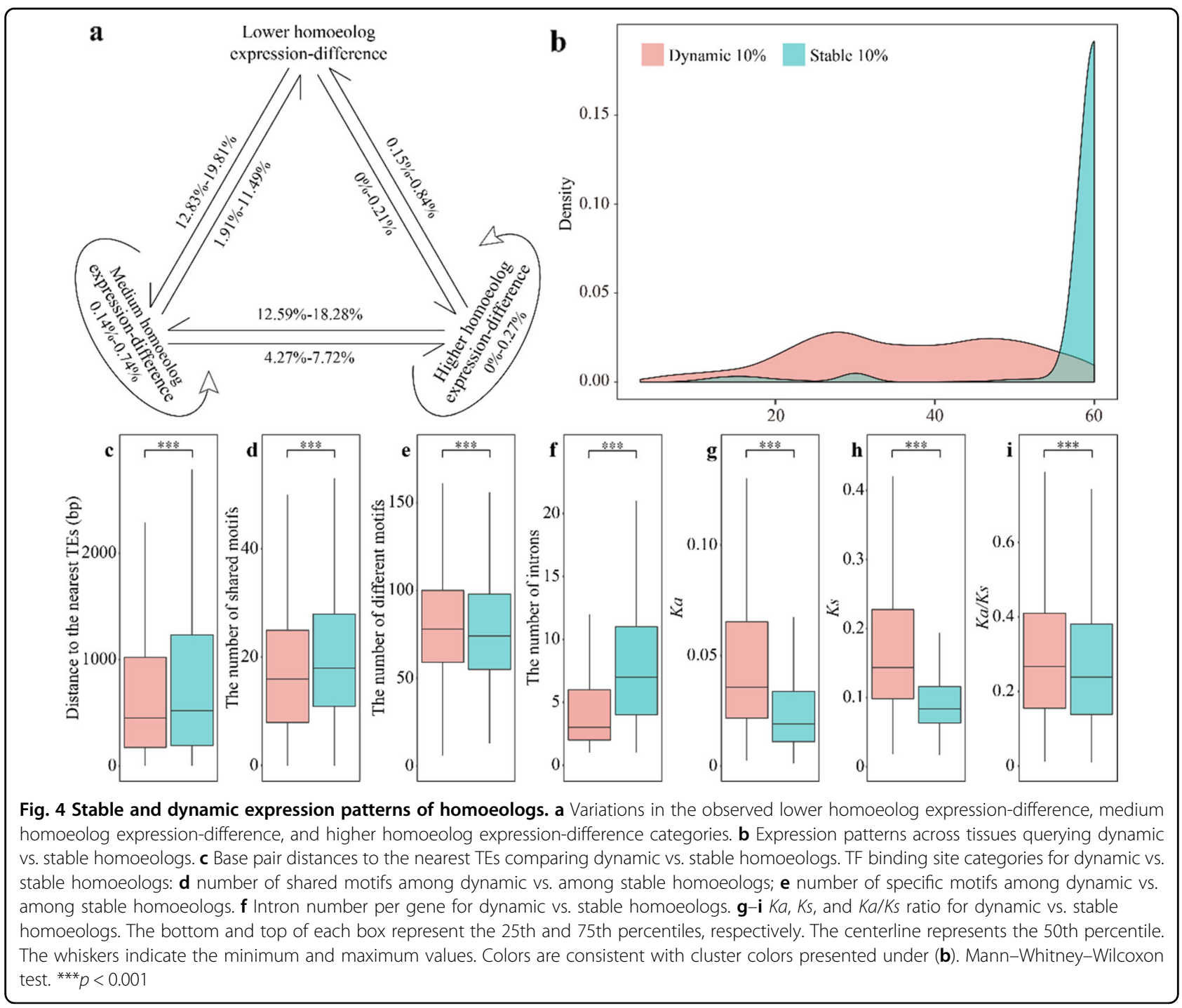


splicing via the spliceosome (Supplementary Table 13). In contrast, dynamic homoeologs were enriched for defense, secondary metabolic processes, and external stimuli responses, functions that more frequently determine fitness differences (Supplementary Table 14). Approximately $47 \%$ of stable homoeologs belonged to the lower homoeolog expression-difference category, whereas twothirds of the dynamic homoeologs were part of the higher homoeolog expression-difference category. Compared to stable homoeologs, dynamic homoeologs were significantly closer to TEs and contained fewer conserved TF binding sites (Fig. 4c-e). We also found that the number of flanking TEs (within $2 \mathrm{~kb}$ ) for dynamic homoeologs was on average higher (1.52 vs. 1.37), and dynamic homoeologs also contained fewer introns than stable homoeologs (Fig. 4f). Dynamic homoeologs had a significantly higher $K a, K s$, and $K a / K s$ ratio, indicating that they have relaxed selection pressure (Fig. $4 \mathrm{~g}-\mathrm{i}$ ).

These results indicate that spatiotemporal expression patterns are positively correlated with differences in flanking TEs, cis-regulatory elements, coding sequences, and selection pressure. These observed changes in spatiotemporal expression patterns, as well as the relaxation of selection pressure, the proximity of TE and the difference of TF binding sites, may lead to functional innovation through sub- or neofunctionalization, following, for instance, divergence of gene expression.

To further examine whether these homoeologs are coordinately expressed, we also constructed a weighted gene coexpression network for the red-flowered and purple-flowered varieties separately. For the redflowered variety, these networks were composed of 26 modules and contained $98 \%$ of all expressed genes. We found $34 \%$ of the homoeologs in the same coexpression module (Supplementary Table 15), while the majority of homoeologs $(66 \%)$ were in different modules. To quantify whether homoeologs in different modules have similar or divergent expression patterns, we calculated the Euclidean distance between module eigengenes. Homoeologs for which the pairwise distance was zero were in the same module. Homoeologs with distances greater than zero were in different modules. When the distance between homoeologs was greater than $50 \%$ of the median distance between eigengenes, the two genes were classified as having "divergent" expression patterns in different modules; otherwise, they had "similar" expression patterns. We found only $33 \%$ of homoeologs with a divergent pattern, suggesting a globally highly coordinated expression pattern (Supplementary Table 15). A similar coordinated expression pattern was also found for the purpleflowered variety (Supplementary Table 15). The "divergent" expression patterns in different modules foreshadow sub- or neofunctionalization between homoeologs ${ }^{34}$.

\section{Discussion}

Plant genomes accommodate much more redundancy, diversity, and dynamics than animal genomes ${ }^{35}$. Owing to their sessile nature, plants are constantly exposed to a multitude of environmental stressors. Such genomic redundancy is often provided as the raw material allowing plants to develop relatively rapid functional innovations ${ }^{36}$. Polyploidization/WGD events, often accompanied by the expansion of large amounts of repeated genes, large genomic rearrangements, and increased genetic variation, are thus considered catalysts for species diversification and evolutionary novelty in plants ${ }^{10,37-40}$. A recently published phylotranscriptomic study presented compelling evidence of widespread ancient polyploidy in Lamiaceae, particularly within the species-rich and chemically diverse Nepetoideae, where S. splendens resides ${ }^{41}$. Our study provides important genomic resources to further understand the genetic and genomic dynamics related to WGD that contribute to specific trait innovations, novelties in specialized metabolism, and subsequent diversification.

Following a polyploidization event, one of the subgenomes, often referred to as the "dominant" subgenome, exhibits significantly higher gene content and greater gene expression than the "recessive" subgenome ${ }^{42}$. There is also growing evidence in polyploid plants for the nonadditive contribution of parental genomes to gene expression $^{43-47}$. At the subgenome level, Thomas et al. ${ }^{42}$ provided details of "biased fractionation" of two progenitor genomes. Findings in cotton also suggested that ancient genomic fractionation persists and can influence the modern cotton genome's functional space, despite approximately 60 million years of evolution ${ }^{48}$. When examining more recent hybridization events, this asymmetric gene expression favoring either one of the A, B, or $D$ cotton subgenomes was absent, and instead, the expression dominance was region- and cell type-dependent, with up to $20-40 \%$ of homoeologous pairs exhibiting expression bias in developmental stages and tissues across endosperm development ${ }^{49,50}$. Similar patterns of localized expression bias were also observed in Brassica napus $^{51}$, as well as in allotetraploid cotton ${ }^{45}$. As shown in Fig. 3a, our results at the subgenome level also suggest an inconsistent, biased expression pattern by resolving the expression biases to the homoeologs. Furthermore, although marginally significant, TE-mediated epigenetic silencing might lead to the repression of gene expression for the dominant subgenome in S. splendens (SAyes_SBno in Fig. $3 b)^{52}$. However, when studying TE adjacency to homoeologs, this TE-mediated silencing effect was found to be more prevalent on "recessive" homoeologous chromosomes (Fig. 3b), suggesting an initial cause of chromosome degradation. As a key driver of mutagenesis in the genome, TEs are capable of inducing initial 
differences in gene expression among homeologs of the polyploid genome ${ }^{52}$. Together with the large numbers of intact, solo, and truncated LTR-RTs identified in this study (Supplementary Fig. 37), we speculate that the ineffective TE removal was attributed to the inconsistent expression biases in the S. splendens genome.

In addition to the WGD shared by most eudicots, we identified three $S$. splendens lineage-specific WGD events. Following each of the WGD events, the doubling of genome copies often leads to two nonmutually exclusive evolutionary scenarios owing to the inflated TE content ${ }^{29,53}$. Originally proposed by Barbara McClintock ${ }^{54}$, the widespread upregulation of TEs as a consequence of "genome shock" would facilitate alterations in gene expression, likely through epigenetic regulation such as DNA methylation, to maintain genome stability ${ }^{55,56}$. Alternatively, WGD may lead to a buffering effect on recessive deleterious mutations and loss-of-function mutations caused by TE insertion. Such relaxed selective pressure could readily promote TE overaccumulation without transposition bursts ${ }^{29}$.

Comparing expression and epigenetic profiles in parental and hybrid contexts, despite the major reorganization of genes and stress-induced TE expression, no evidence for global gene expression dysregulation caused by "genomic shock" can be concluded ${ }^{57}$. As depicted in Fig. 4a, stable, high expression for most of the homoeologs across tissue types was found, regardless of their TE adjacency (Fig. 4c). Conversely, the dynamic homoeologs, as the top $10 \%$ of the homoeologs that exhibited differential expression changes across tissue types, demonstrated a broad range of genetic expression and had significantly higher synonymous, nonsynonymous mutations and, more importantly, significantly relaxed selection pressure (Fig. $4 \mathrm{~g}-\mathrm{i}$ ). Functional analysis of these dynamic homoeologs also implied their characteristics in defense, secondary metabolic processes, and external stress responses (Supplementary Table 14). In the present study, we provided our adaptive evolution viewpoints on the TE-driven functional differentiation of homoeologs in polyploid genomes ${ }^{10,58,59}$. Finally, our results support adaptability in polyploid genomes as a result of relaxed purifying selection on the increased standing variation of environmentally responsive and stress-related genes ${ }^{20,60}$.

In conclusion, our chromosome-scale high-quality assembly of S. splendens and genomic analyses provide essential resources to understand the dynamic evolution following WGD in Lamiales. In addition, our findings will further benefit the functional genomics and molecular breeding of $S$. splendens, such as genome editing practices.

\section{Materials and methods Plant material}

The sequenced individual of S. splendens (Aoyunshenghuo) was collected from the Beijing Institute of
Landscape Architecture germplasm bank. This variety was originally developed by multiple rounds of selfing of one hybrid.

\section{Hi-C library construction and scaffolding}

Young leaves of individual plants from the same S. splendens variety were collected for Hi-C library construction with the Proximo ${ }^{\mathrm{TM}} \mathrm{Hi}-\mathrm{C}$ Plant kit (Phase Genomics) following the manufacturer's protocol. Briefly, fresh-leaf tissue $(0.2 \mathrm{~g})$ was chopped, and chromatin was immediately crosslinked, fragmented, and proximity ligated, followed by library construction. The final library was size selected to contain 300-600 bp fragments and sequenced on the Illumina HiSeq 2500 device under paired-end $150 \mathrm{bp}$ mode. Adapters were trimmed, and low-quality sequences were removed using fastp v0.19.5 ${ }^{61}$. Quality-filtered reads were aligned to the $S$. splendens contigs using bwa v0.7.12 ${ }^{62}$ with strict parameters $(-n 0)$ to prevent mismatches and nonspecific alignments in the Juicer v1.5.6 pipeline $^{63}$. Read pairs were merged, and PCR duplicates were filtered prior to constructing the interaction based on the distance matrix. Chimeras were split, and contigs were ordered and oriented using the 3d-DNA pipeline with default parameters ${ }^{64}$. The resulting $\mathrm{Hi}-\mathrm{C}$ contact matrix was visualized using Juicebox $\mathrm{v} 180922^{65}$, contig misassemblies and scaffold misjoins were manually detected and corrected based on neighboring interactions. In total, this method identified 22 highconfidence clusters representing the haploid chromosome number in S. splendens. The manually validated assembly was used as input to build 22 pseudomolecules using the finalize-output.sh script from 3d-DNA. Chromosomes were renamed and ordered by size, and homoeologous chromosomes were numbered consecutively.

\section{Optimization of genome assembly}

The genome assembly was gap closed twice using LR_Gapcloser v1.1 ${ }^{66}$ with PicBio data. Then, we polished the assembly a third time using pilon $v 1.22^{67}$ with Illumina short reads. Contigs with an identity of more than $99 \%$ were regarded as redundant sequences and were removed. The final assembly of S. splendens was $807 \mathrm{Mb}$.

\section{Repeat annotation}

De novo repeat identification was pursued with RepeatModeler v1.0.10 (http://www.repeatmasker.org/ RepeatModeler/), which employs two complementary computational methods (RECON v1.08 and RepeatScout v1.0.5 ${ }^{68}$ for identifying repeat element boundaries and family relationships from sequence data. The consensus repeat sequences generated above were combined and used for further characterization of TEs with RepeatMasker v4.0.7 (http://www.repeatmasker.org/). 


\section{Gene annotation}

AUGUSTUS v3.2.3 $3^{69,70}$ was employed for ab initio gene prediction. Then, the transcriptome assembly ${ }^{17}$ was aligned to the repeat-masked reference genome assembly with BLAST v2.2.28 $+{ }^{71}$. Next, protein sequences from A. thaliana ${ }^{72}$, S. miltiorrhiza ${ }^{73,74}$, and S. splendens ${ }^{17}$ were aligned to the masked genome assembly with BlastX. After optimization using Exonerate v2.4.0 $0^{75}$, the gene model was finalized for prediction using the MAKER package v2.31.9 ${ }^{76}$ with AUGUSTUS v3.2.3. The quality of gene prediction was assessed using annotation edit distance for each of the predicted genes as part of MAKER. Pseudogenes were identified using Pseudopipe ${ }^{77}$ with default parameters. The programs tRNAScan-SE v2.0.5 ${ }^{78}$ and RNAmmer v1.2 ${ }^{79}$ were used to predict tRNA and rRNA, respectively, and other ncRNAs were identified by searching against the Rfam database (http://eggnogdb. embl.de/). The functions of each gene model were predicted by homoeology searches with BLAT v0.36 ${ }^{80}$ against the UniProt database ${ }^{81}$. Protein annotation against $\mathrm{Pfam}^{82,83}$ and InterProScan v5.27-66.0 ${ }^{84}$ was also conducted using the MAKER package. In addition, we mapped the predicted genes to GO and KEGG classifications.

\section{Centromere/pericentromeric detection}

Putative centromere/pericentromeric regions were predicted based on corrected $\mathrm{Hi}-\mathrm{C}$ data and the tendency of formed clusters in three-dimensional space with a published bioinformatic procedure ${ }^{30}$. The centromeric regions were further delimited by examining the distribution of LINE1.

\section{Full-length LTR-RT annotation}

We used LTRharvest v1.5.8 $8^{85}$ and LTRdigest ${ }^{86}$ for de novo prediction of LTR-RTs. The LTR-RT candidates that possessed complete Gap-Pol protein sequences were retained as intact LTR-RTs $(I)$, while solo-LTRs $(S)$ and truncated LTRs $(T)$ were identified based on sequence similarity to the intact LTR-TRs $(E$ value $<1 \mathrm{e}-10$, overlap length $>90 \%$, identity $>90 \%$ ). Then, LTR homoeology within $15 \mathrm{~kb}$ of sequence data both up- and downstream was extracted and compared with Gap-Pol protein sequences within the rexdb ${ }^{87}$ database using TBLASTN ${ }^{71}$. We considered the corresponding LTRs as truncated LTR-RTs if they had at least 50\% Gag-Pol covered by one side of the flanking sequence and $30 \%$ identity. Lacking Gag-Pol up- and downstream of the LTR was considered to represent a solo-LTR.

\section{Differential proliferation, age dynamics, and gene proximity of different LTR-RT families}

The insertion time of LTR-RTs was estimated according to the difference between the $5^{\prime}$-LTR and $3^{\prime}$-LTR of the same transposon ${ }^{88}$ using MAFFT v7.221 ${ }^{89}$ with a mutation rate of $1.3 \mathrm{e}-8$ substitutions year ${ }^{-1}$ per site. Although the actual pattern of LTR-RT activation and amplification appeared at the family level, as defined by $>80 \%$ sequence homoeology in the LTR-RTs, our focus was on holistic genomic characteristics that can be more carefully dissected and compared at the LTR-RT superfamily level ( $>60 \%$ homoeology). Then, we calculated the distances between intact LTR-RTs and adjacent genes and examined the relationships of proximity to genes and insertion time.

To further understand the relationship among individual LTR-RTs, we aligned the $5^{\prime}$-LTR sequences of all LTRRTs. If two LTRs covered at least $70 \%$ of the length of each other and had at least $60 \%$ identity, they were assigned to the same cluster ${ }^{90}$. We also compared Solo-LTR-RTs and truncated LTR-RTs to the same cluster containing the $5^{\prime}$ LTR from the most similar intact LRT-RTs. The ratios of solo-LTR-RTs and truncated LTR-RTs to intact LTR-RTs (S:I, T:I) and their sum were then evaluated separately to investigate the removal rate of LTR-RTs over the past several million. Furthermore, we evaluated the proportion of clusters with $S: I$ values greater than 3 .

\section{Molecular phylogenetic analysis}

We performed a comparative genomic investigation of S. splendens with A. paniculata ${ }^{91}$, A. majus $^{24}, B$. hygrometrica $^{26}$, Fraxinus excelsior ${ }^{92}$, H. impetiginosus $^{93}$, L. brevidens ${ }^{94}$, M. guttatus ${ }^{22}$, S. miltiorrhiza ${ }^{73}$, S. baicalensis $^{95}$, S. indicum ${ }^{96}, T$. grandis ${ }^{25}$, and $U$. gibba ${ }^{27}$ as representatives of the Lamiales order and Vitis vinifera as the outgroup.

OrthoFinder2 v2.3.1 $1^{97}$ was used to identify homoeologous gene clusters. Based on 373 orthogroups, IQTREE v1.6.7 $7^{98}$ was used to build a phylogenetic tree. MAFFT v7.407 ${ }^{89}$ was used to align homoeologs before transforming aligned protein sequences into codon alignment. The concatenated amino acid sequences were trimmed using trimAL v1.4 ${ }^{99}$ with -gt 0.8 -st 0.001 -cons 60. The program MCMCTree of PAML $v 4.9 \mathrm{~h}^{100}$ was used to estimate the neutral evolutionary rate with an independent substitution rate (clock $=2)$, GTR substitution model, 2.1e6 iterations, and discarded 1e5 iterations as burn-in and fossil date points from TimeTree (http:// timetree.org): 110-124 MYA split time between $V$. vinifera and F. excelsior.

\section{Whole-genome duplication}

Syntenic blocks containing at least five genes were identified using MCScanX $\mathrm{X}^{101}$ with default parameters. KaKs_Calculator $2.0^{102}$ was used to calculate $K a, K s$, and the $K a / K s$ ratio by implementing the $Y N$ model. $K s$ values $>5.0$ were excluded from all analyses due to saturated substitution as synonymous sites ${ }^{103,104}$. 


\section{Homoeolog identification}

We excluded syntenic blocks between nonhomoeologous chromosome pairs and retained only the gene pairs on the syntenic blocks between homoeologous chromosomes as homoeologs.

\section{Structure variation detection}

The Nucmer alignment tool from the MUMmer v4.0.0 toolbox ${ }^{105,106}$ was used to perform whole-genome alignments. Nucmer was run with -maxmatch to obtain all alignments between two homoeologous chromosomes, including -c 500, -b 500, and -1 100 parameters. Subprograms Delta-filter and show-coords were employed to filter the alignments and convert them into tab-delimited files. Finally, SyRI ${ }^{107}$ was used to identify inversions, translocations, duplications, inverted translocations, and inverted duplications. We compared the number of TEs in the upper and lower $2 \mathrm{k}$ regions at the SV breakpoints and 22,000 4k regions (1000 segments per chromosome) randomly selected from the 22 chromosomes using BEDTools v2.29.2 ${ }^{108}$. Similarly, we used BEDTools to compare the intersection of structural variant segments and genes.

\section{Mapping of RNA-seq reads}

Data were collected for three independent biological replicates from five major tissues with two different varieties. Low-quality reads were removed, and the remaining reads were mapped to the genome using hisat2 v2.0.0 ${ }^{109}$. We summarized expression levels from the transcript level to the gene level using featureCounts $v 1.5 .3^{110}$. If the expression value of a gene or homoeolog exceeded 0.5 TPM in any sample, we considered them to be expressed genes or high-confidence homoeologs. Differentially expressed homoeologs were compared using the DESeq2 package $^{111}$.

\section{Dynamic and stable homoeologs identification}

We first identified homoeologs that were differentially expressed $\geq 2(p<0.05)$ in all tissues and pooled tissue. Then, we merged homoeolog sets. For each pair of homoeologs, if $p \geq 0.05$ in a tissue, we defined them as no difference $(\mathrm{FC}=0)$. Dynamic homoeologs were defined as the top $10 \%$ with the largest FC change between different single tissues and pooled tissues, and stable homoeologs were defined as the top $10 \%$ with the smallest FC change.

\section{Transcription factor (TF) and TF binding site identification}

The protein sequence was submitted to plantTFDB ${ }^{112}$ to identify TFs with the best hit in Arabidopsis thaliana. The $2 \mathrm{~kb}$ sequence upstream of the gene was used to identify TF binding sites present in the promoters of genes. The FIMO tool from MEME suite $v 4.12^{113}$ was used with a position weight matrix obtained from
plantTFDB to predict TF binding sites. FIMO was run with a $p$ value threshold of $1 \mathrm{e}-05$, -motif-pseudo of $1 \mathrm{e}-08$ and a -max-stored-scores of $1 \mathrm{e} 6$.

\section{WGCNA network construction}

Coexpression networks were separately built for each of the two varieties with RNA-seq gene expression data from five different tissues using the WGCNA R package ${ }^{114}$. The soft power threshold was calculated as the first power to exceed a scale-free topology fit index of 0.9 for each network separately. Signed hybrid networks were constructed blockwise using the function blockwiseModules. The topographical overlap matrices (TOM) were calculated by the blockwiseModules function using TOMType = "unsigned." The parameter mergeCutHeight $=0.25$ was used to merge similar modules.

\section{GO enrichment analysis}

GO enrichment analysis was performed using the $\mathrm{R}$ package clusterProfiler ${ }^{115}$. The $p$ values were adjusted for multiple comparisons using the method of Benjamini and Hochberg ( $p<0.05$ was considered significant).

\section{Acknowledgements}

This study was supported by the National Natural Science Foundation (31600527), The Fundamental Research Funds for the Central Universities in Beijing Forestry University (2018BLCB08), and the Project of Construction of World Class Universities in Beijing Forestry University (2019XKJS0308). Y. V. d. P. acknowledges funding from the European Research Council (ERC) under the European Union's Horizon 2020 research and innovation program (grant agreement no. 833522) and from Ghent University (Methusalem funding, BOF. MET.2021.0005.01).

\section{Author details}

'Beijing Advanced Innovation Center for Tree Breeding by Molecular Design, National Engineering Laboratory for Tree Breeding, Key Laboratory of Genetics and Breeding in Forest Trees and Ornamental Plants, Ministry of Education, College of Biological Sciences and Technology, Beijing Forestry University, Beijing 100083, China. ${ }^{2}$ Ori (Shandong) Gene Science and Technology Co., Ltd, Weifang 261000 Shandong, China. ${ }^{3}$ Départment des Sciences du Bois et de la Forêt, Faculté de Foresterie, de Géographie et Géomatique, Université Laval, Québec City, QC G1V 0A6, Canada. ${ }^{4}$ Department of Forest and Conservation Sciences, Faculty of Forestry, University of British Columbia, Vancouver, BC V6T 1Z4, Canada. ${ }^{5}$ Department of Ecology and Environmental Science, Umeå Plant Science Centre, Umeå University, SE-901 87 Umeå, Sweden. ${ }^{6}$ Department of Biochemistry and Molecular Biology, 246 Noble Research Center, Oklahoma State University, Stillwater, OK, USA. ${ }^{7}$ Department of Plant Biotechnology and Bioinformatics, Ghent University, Ghent, Belgium. ${ }^{8}$ VIB Center for Plant Systems Biology, 9052 Ghent, Belgium. ${ }^{9}$ Centre for Microbial Ecology and Genomics, Department of Biochemistry, Genetics and Microbiology Genetics, University of Pretoria, Private Bag X20, Pretoria 0028, South Africa. ${ }^{10}$ College of Horticulture, Academy for Advanced Interdisciplinary Studies, Nanjing Agricultural University, Nanjing 210095, China

\section{Author contributions}

J.-F. M. and W. Z. conceived and designed the study; K. H. J., H. L., R. G. Z., S. S. Z., S. Q. J., X. M. Y., X. C. T., Z. C. L., H. L., T. L. S., J. X., S. N., J. F. G., Y. T. B., and $W$. Z. prepared the materials, conducted the experiments, analyzed the data and prepared the results; K. H. J. and J. F. M. wrote the manuscript; Y. V. d. P. C. C., I. P., Y. A. E., X. R. W., and W. Z. edited and improved the manuscript; all authors approved the final manuscript. 


\section{Data availability}

The Whole Genome Shotgun project has been deposited at DDBJ/ENA/ GenBank under the accession PNBA00000000. The version described in this paper is PNBA00000000. The raw sequence data have been deposited in the Short Read Archive under NCBI BioProject ID PRJNA422035.

\section{Conflict of interest}

The authors declare no competing interests.

Supplementary information The online version contains supplementary material available at https://doi.org/10.1038/s41438-021-00614-y.

Received: 7 February 2021 Revised: 12 May 2021 Accepted: 20 May 2021 Published online: 01 September 2021

\section{References}

1. Harley, R. M. et al. Labiatae. Flowering Plants Dicotyledons 167-275 (Springer, 2004).

2. Palomino, G., Mercado, P. \& TP, R. Chromosomes of Salvia subgenus Calosphace (Lamiaceae), a preliminary report. Cytologia 51, 381-386 (1986).

3. Alberto, C. M., Sanso, A. M. \& Xifreda, C. C. Chromosomal studies in species of Salvia (Lamiaceae) from Argentina. Bot. J. Linn. Soc. 141, 483-490 (2003).

4. Regnault-Roger, $C$. The potential of botanical essential oils for insect pest control. Integr. Pest Manag. Rev. 2, 25-34 (1997).

5. Joyce, A. The new book of Salvias: sages for every garden. Booklist 99 , 1263-1263 (2003).

6. Mandáková, T. \& Lysak, M. A. Post-polyploid diploidization and diversification through dysploid changes. Curr. Opin. Plant Biol. 42, 55-65 (2018).

7. Jiao, Y. et al. Ancestral polyploidy in seed plants and angiosperms. Nature 473, 97 (2011)

8. Soltis, P. S. Ancient and recent polyploidy in angiosperms. N. Phytol. 166, 5-8 (2005).

9. Doyle, J. J. et al. Evolutionary genetics of genome merger and doubling in plants. Annu. Rev. Genet. 42, 443-461 (2008).

10. Van de Peer, Y., Mizrachi, E. \& Marchal, K. The evolutionary significance of polyploidy. Nat. Rev. Genet. 18, 411 (2017).

11. Wendel, J. F. Genome evolution in polyploids. Plant Molecular Evolution 225-249 (Springer, 2000)

12. Soltis, P. S. \& Soltis, D. E. The role of hybridization in plant speciation. Annu. Rev. Plant Biol. 60, 561-588 (2009).

13. Van de Peer, Y., Ashman, T. L., Soltis, P. S. \& Soltis, D. E. Polyploidy: an evolutionary and ecological force in stressful times. The Plant Cell 33, 11-26 (2021).

14. Makova, K. D. \& Li, W. H. Divergence in the spatial pattern of gene expression between human duplicate genes. Genome Res. 13, 1638-1645 (2003).

15. Innan, H. \& Kondrashov, F. The evolution of gene duplications: classifying and distinguishing between models. Nat. Rev. Genet. 11, 97 (2010).

16. Kondrashov, F. A. Gene duplication as a mechanism of genomic adaptation to a changing environment. Proc. R. Soc. B. 279, 5048-5057 (2012).

17. Dong, A. X. et al. High-quality assembly of the reference genome for scarlet sage, Salvia splendens, an economically important ornamental plant. GigaScience 7, giy068 (2018).

18. Simão, F. A., Waterhouse, R. M., loannidis, P., Kriventseva, E. V. \& Zdobnov, E. M. BUSCO: assessing genome assembly and annotation completeness with single-copy orthologs. Bioinformatics 31, 3210-3212 (2015).

19. Ou, S., Chen, J. \& Jiang, N. Assessing genome assembly quality using the LTR Assembly Index (LAl). Nucleic Acids Res. 46, e126 (2018).

20. Otto, S. P. The evolutionary consequences of polyploidy. Cell 131, 452-462 (2007).

21. Murat, F., Armero, A., Pont, C., Klopp, C. \& Salse, J. Reconstructing the genome of the most recent common ancestor of flowering plants. Nat. Genet. 49, 490 (2017).

22. Hellsten, $U$. et al. Fine-scale variation in meiotic recombination in Mimulus inferred from population shotgun sequencing. Proc. Natl Acad. Sci. USA 110, 19478-19482 (2013).

23. Zhang, $\mathrm{H}$. et al. Genome sequencing of the important oilseed crop Sesamum indicum L. Genome Biol. 14, 401 (2013).
24. Li, M. et al. Genome structure and evolution of Antirrhinum majus L. Nat. Plants 5, 174 (2019).

25. Zhao, D. et al. A chromosomal-scale genome assembly of Tectona grandis reveals the importance of tandem gene duplication and enables discovery of genes in natural product biosynthetic pathways. GigaScience 8, giz005 (2019).

26. Xiao, L. et al. The resurrection genome of Boea hygrometrica: a blueprint for survival of dehydration. Proc. Natl Acad. Sci. USA 112, 5833-5837 (2015).

27. Ibarra-Laclette, E. et al. Architecture and evolution of a minute plant genome. Nature 498, 94 (2013).

28. Song, Z. et al. A high-quality reference genome sequence of Salvia miltiorrhiza provides insights into tanshinone synthesis in its red rhizomes. Plant Genome 13, e20041 (2020).

29. Baduel, P., Quadrana, L., Hunter, B., Bomblies, K. \& Colot, V. Relaxed purifying selection in autopolyploids drives transposable element over-accumulation which provides variants for local adaptation. Nat. Commun. 10, 1-10 (2019).

30. Marie-Nelly, $\mathrm{H}$. et al. Filling annotation gaps in yeast genomes using genome-wide contact maps. Bioinformatics 30, 2105-2113 (2014).

31. Piazza, A. \& Heyer, W. D. Homologous recombination and the formation of complex genomic rearrangements. Trends Cell Biol. 29, 135-149 (2019).

32. Lynch, M. \& Conery, J. S. The evolutionary fate and consequences of duplicate genes. Science 290, 1151-1155 (2000).

33. Kondrashov, F. A., Rogozin, I. B., Wolf, Y. I. \& Koonin, E. V. Selection in the evolution of gene duplications. Genome Biol. 3, 1-9 (2002).

34. De Smet, R., Sabaghian, E., Li, Z., Saeys, Y., \& Van de Peer, Y. Coordinated functional divergence of genes after genome duplication in Arabidopsis thaliana. Plant Cell 29, 2786-2800 (2017).

35. Kejnovsky, E., Leitch, I. J. \& Leitch, A. R. Contrasting evolutionary dynamics between angiosperm and mammalian genomes. Trends Ecol. Evol. 24, 572-582 (2009).

36. Murat, F. et al. Ancestral grass karyotype reconstruction unravels new mechanisms of genome shuffling as a source of plant evolution. Genome Res. 20, 1545-1557 (2010).

37. Soltis, P. S., Marchant, D. B., Van de Peer, Y. \& Soltis, D. E. Polyploidy and genome evolution in plants. Curr. Opin. Genet. Dev. 35, 119-125 (2015).

38. Schranz, M. E., Mohammadin, S. \& Edger, P. P. Ancient whole genome duplications, novelty and diversification: the WGD Radiation Lag-Time Model. Curr. Opin. Plant Biol. 15, 147-153 (2012).

39. Tank, D. C. et al. Nested radiations and the pulse of angiosperm diversification: increased diversification rates often follow whole genome duplications. N. Phytol. 207, 454-467 (2015).

40. Soltis, P. S. \& Soltis, D. E. Ancient WGD events as drivers of key innovations in angiosperms. Curr. Opin. Plant Biol. 30, 159-165 (2016).

41. Godden, G. T., Kinser, T. J., Soltis, P. S. \& Soltis, D. E. Phylotranscriptomic analyses reveal asymmetrical gene duplication dynamics and signatures of ancient polyploidy in mints. Genome Biol. Evol. 11, 3393-3408 (2019).

42. Thomas, B. C., Pedersen, B. \& Freeling, M. Following tetraploidy in an Arabidopsis ancestor, genes were removed preferentially from one homeolog leaving clusters enriched in dose-sensitive genes. Genome Res. 16, 934-946 (2006).

43. Schnable, J. C., Springer, N. M. \& Freeling, M. Differentiation of the maize subgenomes by genome dominance and both ancient and ongoing gene loss. Proc. Natl Acad. Sci. USA 108, 4069-4074 (2011).

44. Ramirez-Gonzalez, R. H. et al. The transcriptional landscape of polyploid wheat. Science 361, eaar6089 (2018).

45. Yoo, M., Szadkowski, E. \& Wendel, J. Homoeolog expression bias and expression level dominance in allopolyploid cotton. Heredity 110, 171-180 (2013).

46. Bottani, S., Zabet, N. R., Wendel, J. F. \& Veitia, R. A. Gene expression dominance in allopolyploids: hypotheses and models. Trends Plant Sci. 23 393-402 (2018).

47. Bird, K. A., VanBuren, R., Puzey, J. R. \& Edger, P. P. The causes and consequences of subgenome dominance in hybrids and recent polyploids. $N$. Phytol. 220, 87-93 (2018).

48. Renny-Byfield, S., Gong, L., Gallagher, J. P. \& Wendel, J. F. Persistence of subgenomes in paleopolyploid cotton after 60 my of evolution. Mol. Biol. Evol. 32, 1063-1071 (2015)

49. Pfeifer, M. et al. Genome interplay in the grain transcriptome of hexaploid bread wheat. Science 345, 1250091 (2014).

50. Harper, A. L. et al. Genome distribution of differential homoeologue contributions to leaf gene expression in bread wheat. Plant Biotechnol. J. 14, 1207-1214 (2016). 
51. Chalhoub, B. et al. Early allopolyploid evolution in the post-Neolithic Brassica napus oilseed genome. Science 345, 950-953 (2014).

52. Hollister, J. D. \& Gaut, B. S. Epigenetic silencing of transposable elements: a trade-off between reduced transposition and deleterious effects on neighboring gene expression. Genome Res. 19, 1419-1428 (2009).

53. Vicient, C. M. \& Casacuberta, J. M. Impact of transposable elements on polyploid plant genomes. Ann. Bot. 120, 195-207 (2017).

54. McClintock, B. Nobel lecture: the significance of response of the genome to challenge. Science 226, 792-801 (1984).

55. Lafon-Placette, C. \& Köhler, C. Epigenetic mechanisms of postzygotic reproductive isolation in plants. Curr. Opin. Plant Biol. 23, 39-44 (2015).

56. Zhang, M. et al. Transcriptome analysis reveals hybridization-induced genome shock in an interspecific F1 hybrid from Camellia. Genome 61, 477-485 (2018).

57. Göbel, U. et al. Robustness of transposable element regulation but no genomic shock observed in interspecific Arabidopsis hybrids. Genome Biol. Evol. 10, 1403-1415 (2018).

58. Feldman, M., Levy, A. A., Fahima, T. \& Korol, A. Genomic asymmetry in allopolyploid plants: wheat as a model. J. Exp. Bot. 63, 5045-5059 (2012).

59. Ohno, S. Evolution by Gene Duplication (Springer Science \& Business Media, 2013).

60. Coward, J. \& Harding, A. Size does matter: why polyploid tumor cells are critical drug targets in the war on cancer. Front. Oncol. 4, 123 (2014).

61. Chen, S., Zhou, Y., Chen, Y. \& Gu, J. fastp: an ultra-fast all-in-one FASTQ preprocessor. Bioinformatics 34, i884-i890 (2018).

62. $\mathrm{Li}, \mathrm{H}$. Aligning sequence reads, clone sequences and assembly contigs with BWA-MEM. arXiv 1303 (2013).

63. Durand, N. C. et al. Juicer provides a one-click system for analyzing loopresolution Hi-C experiments. Cell Syst. 3, 95-98 (2016).

64. Dudchenko, O. et al. De novo assembly of the Aedes aegypti genome using $\mathrm{Hi}-\mathrm{C}$ yields chromosome-length scaffolds. Science 356, 92-95 (2017).

65. Durand, N. C. et al. Juicebox provides a visualization system for Hi-C contact maps with unlimited zoom. Cell Syst. 3, 99-101 (2016).

66. Xu, G. C. et al. LR_Gapcloser: a tiling path-based gap closer that uses long reads to complete genome assembly. Gigascience 8, giy157 (2018).

67. Walker, B. J. et al. Pilon: an integrated tool for comprehensive microbial variant detection and genome assembly improvement. PloS One 9, e112963 (2014).

68. Price, A. L., Jones, N. C. \& Pevzner, P. A. De novo identification of repeat families in large genomes. Bioinformatics 21, i351-i358 (2005).

69. Keller, O., Kollmar, M., Stanke, M. \& Waack, S. A novel hybrid gene prediction method employing protein multiple sequence alignments. Bioinformatics $\mathbf{2 7}$ 757-763 (2011).

70. Stanke, M., Diekhans, M., Baertsch, R. \& Haussler, D. Using native and syntenically mapped CDNA alignments to improve de novo gene finding. Bioinformatics 24, 637-644 (2008).

71. Boratyn, G. M. et al. Domain enhanced lookup time accelerated BLAST. Biol. Direct 7, 12 (2012)

72. Swarbreck, D. et al. The Arabidopsis Information Resource (TAIR): gene structure and function annotation. Nucleic Acids Res. 36, D1009-D1014 (2007).

73. Zhang, G. et al. Hybrid de novo genome assembly of the Chinese herbal plant danshen (Salvia miltiorrhiza Bunge). GigaScience 4, 62 (2015).

74. $\mathrm{Xu}, \mathrm{H}$. et al. Analysis of the genome sequence of the medicinal plant Salvia miltiorrhiza. Mol. Plant 9, 949-952 (2016).

75. Slater, G. S. C. \& Birney, E. Automated generation of heuristics for biological sequence comparison. BMC Bioinform. 6, 31 (2005)

76. Cantarel, B. L. et al. MAKER: an easy-to-use annotation pipeline designed for emerging model organism genomes. Genome Res. 18, 188-196 (2008).

77. Zhang, Z. et al. PseudoPipe: an automated pseudogene identification pipeline. Bioinformatics 22, 1437-1439 (2006).

78. Lowe, T. M. \& Eddy, S. R. tRNAscan-SE: a program for improved detection of transfer RNA genes in genomic sequence. Nucleic Acids Res. 25, 955-964 (1997).

79. Lagesen, $\mathrm{K}$. et al. RNAmmer: consistent and rapid annotation of ribosomal RNA genes. Nucleic Acids Res. 35, 3100-3108 (2007).

80. Kent, W. J. BLAT-the BLAST-like alignment tool. Genome Res. 12, 656-664 (2002).

81. Bairoch, A. \& Apweiler, R. The SWISS-PROT protein sequence database and its supplement TrEMBL in 2000. Nucleic Acids Res. 28, 45-48 (2000).
82. Punta, M. et al. The Pfam protein families database. Nucleic Acids Res. 40 D290-D301 (2012)

83. Bateman, A. et al. The Pfam protein families database. Nucleic Acids Res. $\mathbf{3 0}$ 276-280 (2002).

84. Quevillon, E. et al. InterProScan: protein domains identifier. Nucleic Acids Res. 33, W116-W120 (2005)

85. Ellinghaus, D., Kurtz, S. \& Willhoeft, U. LTRharvest, an efficient and flexible software for de novo detection of LTR retrotransposons. BMC Bioinform. 9, 18 (2008).

86. Steinbiss, S., Willhoeft, U., Gremme, G. \& Kurtz, S. Fine-grained annotation and classification of de novo predicted LTR retrotransposons. Nucleic Acids Res. 37, 7002-7013 (2009).

87. Neumann, P., Novák, P., Hoštáková, N. \& Macas, J. Systematic survey of plant LTR-retrotransposons elucidates phylogenetic relationships of their polyprotein domains and provides a reference for element classification. Mob. DNA 10, 1 (2019).

88. SanMiguel, P., Gaut, B. S., Tikhonov, A., Nakajima, Y. \& Bennetzen, J. L. The paleontology of intergene retrotransposons of maize. Nat. Genet. 20, 43-45 (1998).

89. Katoh, K. \& Standley, D. M. MAFFT multiple sequence alignment software version 7: improvements in performance and usability. Mol. Biol. Evol. 30 772-780 (2013).

90. Miele, V., Penel, S. \& Duret, L. Ultra-fast sequence clustering from similarity networks with SiLiX. BMC Bioinform. 12, 116 (2011).

91. Sun, W. et al. The genome of the medicinal plant Andrographis paniculata provides insight into the biosynthesis of the bioactive diterpenoid neoandrographolide. Plant J. 97, 841-857 (2019).

92. Sollars, E. S. et al. Genome sequence and genetic diversity of European ash trees. Nature 541, 212 (2017).

93. Silva-Junior, O. B., Grattapaglia, D., Novaes, E. \& Collevatti, R. G. Genome assembly of the Pink Ipê (Handroanthus impetiginosus, Bignoniaceae), a highly valued, ecologically keystone Neotropical timber forest tree. GigaScience 7, gix125 (2017).

94. VanBuren, R. et al. Desiccation tolerance evolved through gene duplication and network rewiring in Lindernia. Plant Cell 30, 2943-2958 (2018).

95. Zhao, Q. et al. The reference genome sequence of Scutellaria baicalensis provides insights into the evolution of wogonin biosynthesis. Mol. Plant 12 935-950 (2019).

96. Wang, L. et al. Genome sequencing of the high oil crop sesame provides insight into oil biosynthesis. Genome Biol. 15, 1-13 (2014).

97. Emms, D. M. \& Kelly, S. OrthoFinder: phylogenetic orthology inference for comparative genomics. Genome Biol. 20, 1-14 (2019).

98. Nguyen, L. T., Schmidt, H. A., von Haeseler, A. \& Minh, B. Q. IQ-TREE: a fast and effective stochastic algorithm for estimating maximum-likelihood phylogenies. Mol. Biol. Evol. 32, 268-274 (2015).

99. Capella-Gutiérrez, S., Silla-Martínez, J. M. \& Gabaldón, T. trimAl: a tool for automated alignment trimming in large-scale phylogenetic analyses. Bioinformatics 25, 1972-1973 (2009).

100. Yang, Z. PAML 4: phylogenetic analysis by maximum likelihood. Mol. Biol. Evol. 24, 1586-1591 (2007)

101. Wang, Y. P. et al. MCScanX: a toolkit for detection and evolutionary analysis of gene synteny and collinearity. Nucleic Acids Res. 40, e49 (2012).

102. Wang, D., Zhang, Y., Zhang, Z., Zhu, J. \& Yu, J. KaKs Calculator 2.0: a toolkit incorporating gamma-series methods and sliding window strategies. Genomics, Proteom. Bioinforma. 8, 77-80 (2010).

103. Vanneste, K., Van de Peer, Y. \& Maere, S. Inference of genome duplications from age distributions revisited. Mol. Biol. Evol. 30, 177-190 (2012).

104. Li, Z. et al. Gene duplicability of core genes is highly consistent across all angiosperms. Plant Cell 28, 326-344 (2016).

105. Marçais, G. et al. MUMmer4: a fast and versatile genome alignment system. PLoS Comp. Biol. 14, e1005944 (2018).

106. Kurtz, S. et al. Versatile and open software for comparing large genomes. Genome Biol. 5, R12 (2004).

107. Goel, M., Sun, H., Jiao, W. B. \& Schneeberger, K. SyRl: finding genomic rearrangements and local sequence differences from whole-genome assemblies. Genome Biol. 20, 277 (2019).

108. Quinlan, A. R. \& Hall, I. M. BEDTools: a flexible suite of utilities for comparing genomic features. Bioinformatics 26, 841-842 (2010).

109. Kim, D., Langmead, B. \& Salzberg, S. L. HISAT: a fast spliced aligner with low memory requirements. Nat. Methods 12, 357-360 (2015). 
110. Liao, Y., Smyth, G. K. \& Shi, W. featureCounts: an efficient general purpose program for assigning sequence reads to genomic features. Bioinformatics 30, 923-930 (2013)

111. Love, M. I., Huber, W. \& Anders, S. Moderated estimation of fold change and dispersion for RNA-seq data with DESeq2. Genome Biol. 15, 550 (2014).

112. Jin, J. et al. PlantTFDB 4.0: toward a central hub for transcription factors and regulatory interactions in plants. Nucleic Acids Res. 4, D1040-D1045 (2016).
113. Grant, C. E., Bailey, T. L. \& Noble, W. S. FIMO: scanning for occurrences of a given motif. Bioinformatics 27, 1017-1018 (2011).

114. Langfelder, P. \& Horvath, S. WGCNA: an R package for weighted correlation network analysis. BMC Bioinforma. 9, 559 (2008).

115. Yu, G., Wang, L. G., Han, Y. \& He, Q. clusterProfiler: an R package for comparing biological themes among gene clusters. Omics 16, 284-287 (2012). 